\title{
Truncated neurokinin-1 receptor is an ubiquitous antitumor target in hepatoblastoma, and its expression is independent of tumor biology and stage
}

\author{
AGNÈS GARNIER ${ }^{1}$, MATTHIAS ILMER ${ }^{2}$, KRISTINA BECKER ${ }^{1}$, BEATE HÄBERLE ${ }^{1}$, \\ DIETRICH VON SCHWEINITZ ${ }^{1}$, ROLAND KAPPLER ${ }^{1}$ and MICHAEL BERGER ${ }^{1}$
}

\author{
${ }^{1}$ Department of Pediatric Surgery, Dr von Hauner Children's Hospital, Ludwig Maximilian University of Munich, \\ D-80337 Munich; ${ }^{2}$ Department of General, Visceral, Transplantation, Vascular and Thoracic Surgery, \\ Hospital of the Ludwig Maximilian University of Munich, D-80336 Munich, Germany
}

Received April 3,2015; Accepted August 25, 2015

DOI: $10.3892 / \mathrm{ol} .2015 .3951$

\begin{abstract}
The substance P (SP; also known as TACl)/neurokinin-1 receptor (NK1R; also known as TACR1) complex is a critical part in the development of cancer. Therefore, NK1R antagonists, such as the clinical drug aprepitant, are currently under investigation as future anticancer agents. In a previous study, NK1R (TACRl) was identified as a potent anticancer target in hepatoblastoma (HB). However, little is known regarding the exact distribution of this target among HB subsets and whether it correlates with clinical features and prognosis. In the present study, mRNA was isolated from 47 children with HB, and reverse transcription-quantitative polymerase chain reaction was performed on the samples to analyze the expression of full-length-TACRl (fl-TACRl) and truncated-TACRl (tr-TACRl). These data were correlated with data obtained from 9 tumor-free controls, as well as with the presence of metastasis, PRETEXT, vascular invasion, histology, age of diagnosis, multifocality, CTNNB1 mutation, gender and overall survival. Additionally, the present study investigated a recently described 16-gene signature characterizing HB known to correlate with prognosis. Compared with tumor-free liver tissue, tumorous tissue expressed TACRI significantly higher for the truncated version $(\mathrm{P}=0.0301)$, and by trend also for the full-length version. Accordingly, the expression of fl-TACRl correlated with the expression of the truncated version $(\mathrm{P}=0.0074)$. Furthermore, a low expression of fl-TACRI correlated with characteristics of the 16-gene signature known to predict prognosis $(\mathrm{P}=0.0222)$. However, there was no correlation between tr-TACRI and the tumor characteristics investigated,
\end{abstract}

Correspondence to: Dr Michael Berger, Department of Pediatric Surgery, Dr von Hauner Children's Hospital, Ludwig Maximilian University of Munich, 4 Lindwurmstrasse, D-80337 Munich, Germany

E-mail: michael.fabian.berger@gmail.com

Key words: neurokinin-1 receptor, aprepitant, substance $\mathrm{P}$, antitumor target, apoptosis, hepatoblastoma including outcome, although a clear trend was observed for some tumor characteristics. The current results reinforced the previously described findings that in $\mathrm{HB}, \mathrm{tr}-T A C R \mathrm{l}$ is overexpressed compared with tumor-free liver tissue. Furthermore, to the best of our knowledge, the present study demonstrated for the first time that tr-TACRl is expressed ubiquitously among the different subsets of HB. Therefore, NK1R may serve as a potent anticancer target in a large variety of patients with $\mathrm{HB}$, independent of tumor biology and clinical stage.

\section{Introduction}

Hepatoblastoma (HB) is the most common liver tumor of childhood (1). If complete surgical resection of the tumor is achieved, the prognosis of children with HB is favorable, with or without additional chemotherapy (standard-risk patients) (1). Despite recent advances in therapy for these children, prognosis remains poor for high-risk patients $(1,2)$. Among high-risk children, chemotherapy is crucial in addition to surgical therapy. However, multi-drug resistance to chemotherapy significantly limits the ability to successfully treat these patients $(1,3)$. Therefore, the employment of novel anticancer agents against $\mathrm{HB}$ is needed.

The use of neurokinin-1 receptor (NK1R; also known as TACR 1) antagonists is a novel and promising approach for future anticancer strategies (4). The peptide substance P (SP; also known as TAC1) is a widely distributed neuronal transmitter that, after binding specifically to NK1R, triggers a broad variety of functions (5). It is known that SP can induce tumor cell proliferation, angiogenesis and migration via NK1R, and that the SP/NK1R complex is an integral part of the cancer cell itself, as well as its tumor microenvironment (6). Aprepitant, a non-peptide NK1R antagonist, is a clinical agent approved by the Food and Drug Administration for the treatment of chemotherapy-induced nausea and vomiting. Its effects as an anticancer agent have been described extensively in vitro and in vivo (6-11). Notably, evidence indicates that it has limited toxic side effects even when administered in high doses $(6,12)$.

In a previous study, we described that TACR1 is highly expressed in human HB, predominantly in its truncated form 
[truncated-TACRI (tr-TACR1)] (8). Compared with full-length (fl-TACR1), tr-NK1R lacks 96 amino acids at the cytoplasmic $\mathrm{C}$-terminus of the receptor that are responsible for intracellular signal transduction. Although this splice variant is considered to be able to couple $\mathrm{G}$ proteins, it exhibits decreased efficiency with respect to internalization and desensitization $(8,13-15)$. The net result of this is a decreased ability for negative feedback inhibition, allowing constant activation despite saturation of the receptor complex (13-15). This, in turn, may contribute to the correlation of expression of this particular splice variant with cancer. In an experimental HB setting, NK1R antagonists acted as highly active anticancer agents in vitro and in vivo, and functioned synergistically with established chemotherapy agents in vitro (8).

In addition to these findings, a molecular 16-gene signature has been described for HB, in order to better classify molecular patterns and biological characteristics of these tumors $(16,17)$. Using this signature, two tumor subclasses resembling distinct phases of liver development can be identified. Notably, this signature discriminates invasive and metastatic from localized HB and predicts prognosis with high accuracy (16). Additionally, it has recently been suggested that the expression of TACR I may correlate with a clinically worse prognosis in some cancers (18-22). However, scientific evidence for such an association remains scarce. No study has previously focused on the expression of TACRI and a possible association with the clinical prognosis in HB. Therefore, the present study analyzed the expression pattern of this target among human $\mathrm{HB}$ subsets and investigated whether it correlates with clinical characteristics, such as stage, biology and outcome, including a 16-gene molecular signature known to correlate with prognosis in these tumors. The current results showed that tr-TACR1 is overexpressed compared with tumor-free liver tissue in HB. Addtionally, tr-TACR 1 was expressed ubiquitously among the different subsets of HB. Therefore, NK1R may serve as a potent anticancer target in a number of patients with $\mathrm{HB}$, independent of tumor biology and clinical stage.

\section{Patients and methods}

Patients and tumor tissues. Analysis of tumor tissue samples from patients with $\mathrm{HB}(\mathrm{n}=47)$ who were all part of the German Cooperative Pediatric Liver Tumor Registry Study HB99 and its subsequent Register for Pediatric Liver Tumors was performed. The two registries were multicentric and were initiated by the German Society for Pediatric Oncology and Hematology. They were open to registration for patients from Germany, Austria and Switzerland up to the age of 20 years with untreated HB. The registry protocols were assigned by the institutional Ethical Committee of the University Children's Hospital Basel (Basel, Switzerland) and the University of Bonn (Bonn, Germany), and written consent was obtained from the parents for treatment, data collection and analysis.

Clinical information, including demographic, therapeutic, tumor and clinical outcome variables, were retrieved from the two clinical studies. The treatment protocol consisted of preoperative chemotherapy followed by delayed surgery and postoperative chemotherapy according to two risk groups (standard- versus high-risk). The two risk groups were based on the International Childhood Liver Tumor Strategy
Group risk criteria (23). Standard risk patients received two or three courses of neoadjuvant ifosfamide, cisplatin and doxorubicin (IPA) chemotherapy prior to surgery ( $1 \mathrm{~g} / \mathrm{m}^{2}$ ifosfamide every $72 \mathrm{~h}$, days $1-3 ; 20 \mathrm{mg} / \mathrm{m}^{2}$ cisplatin every $1 \mathrm{~h}$, days $4-8$; and $60 \mathrm{mg} / \mathrm{m}^{2}$ doxorubicin every $48 \mathrm{~h}$, days 9-10). Radical surgery was conducted after the second or third course depending on the resectability. Postoperatively, another course of IPA was applied. In case of microscopically incomplete resection, two adjuvant courses of IPA were administered. Patients with small-extended tumors (PRETEXT stage I) (24) could be resected without neoadjuvant chemotherapy and were treated with two courses of IPA postoperatively. High-risk patients received up to seven courses of carboplatin-based chemotherapy preoperatively depending on tumor shrinkage and resectability. Patients were initially treated with two courses of carbo/VP16 (200 mg/m² carboplatin every $24 \mathrm{~h}$, days 1-4; and $100 \mathrm{mg} / \mathrm{m}^{2}$ etoposide every $24 \mathrm{~h}$, days $\left.1-4\right)$ followed by stem cell collection. In case of tumor response, the therapy was continued with high dose carboplatin and etoposide (500 mg/m ${ }^{2}$ per $24 \mathrm{~h}$, days $8-5$ ) following autologous stem cell transplantation. Patients without tumor response were treated with IPA. Resection was scheduled as soon as the tumor was determined to be completely resectable. In case of persisting non-resectability, liver transplantation was recommended. Lung metastases were resected if residual metastases were still observed on radiological images after chemotherapy.

Tumor specimens were reviewed by the local institution as well as the Institute of Pediatric Pathology, University of Kiel (Kiel, Germany), which served as a reference center. Matched adjacent liver tissue samples from the surgical specimens without macroscopic or microscopic tumors served as tumor-free controls $(n=9)$. Clinical and molecular data, such as gender, age at diagnosis, PRETEXT staging (24), including vascular invasion and multifocality, metastatic disease, histology, CTNNB1 mutation, 16-gene signature and overall survival, were retrieved from the HB99 database and our recent exome sequencing study, respectively (25).

RNA extraction and reverse transcription. RNA extraction, complementary (c)DNA synthesis and quantitative polymerase chain reaction (qPCR) analysis were performed as previously described (26). Briefly, total RNA was isolated from all the samples using TRIzol ${ }^{\circledR}$ reagent (Invitrogen Life Technologies, Carlsbad, CA, USA) and dissolved in RNase-free water. The purity and quality of the RNA was checked using a Nanodrop ${ }^{\circledR} 2000$ spectrophotometer (Thermo Fisher Scientific, Inc., Waltham, MA, USA). RNA (2 $\mu \mathrm{g})$ was reverse transcribed using SuperScript ${ }^{\mathrm{TM}}$ II reverse transcriptase (Invitrogen Life Technologies), according to the manufacturer's instructions. The amplification reactions were performed with $40 \mathrm{ng}$ complementary DNA, $500 \mathrm{nM}$ forward and reverse primers and iTaq SYBR ${ }^{\circledR}$-Green Supermix (Bio-Rad Laboratories, Hercules, CA, USA) in a final reaction volume of $20 \mu \mathrm{l}$ and were incubated at $95^{\circ} \mathrm{C}$ for $7 \mathrm{~min}$, subjected to 40 cycles of $95^{\circ} \mathrm{C}$ for $30 \mathrm{sec}, 60^{\circ} \mathrm{C}$ for $30 \mathrm{sec}$ and $72^{\circ} \mathrm{C}$ for $30 \mathrm{sec}$, followed by a final extension cycle at $72^{\circ} \mathrm{C}$ for $7 \mathrm{~min}$. All the reactions were conducted on ice to minimize the risk of RNA degradation. cDNA obtained was stored at $-80^{\circ} \mathrm{C}$. 

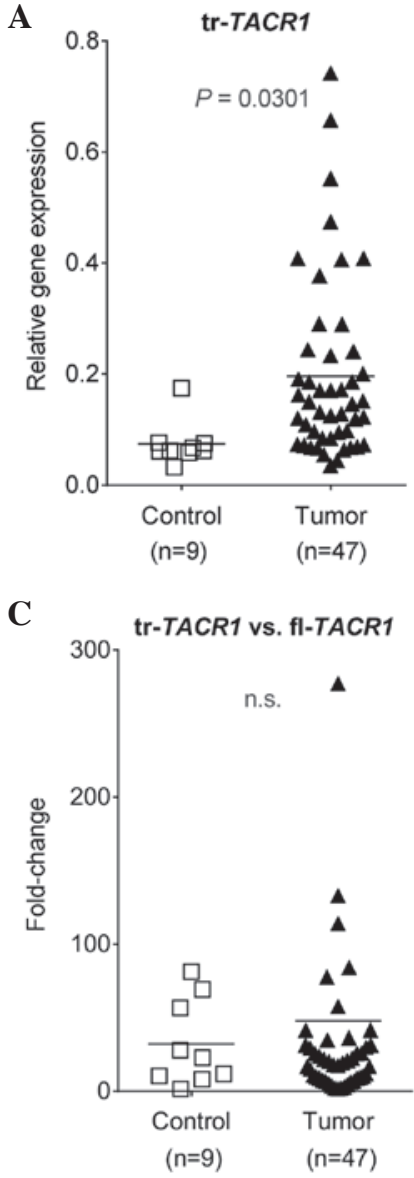

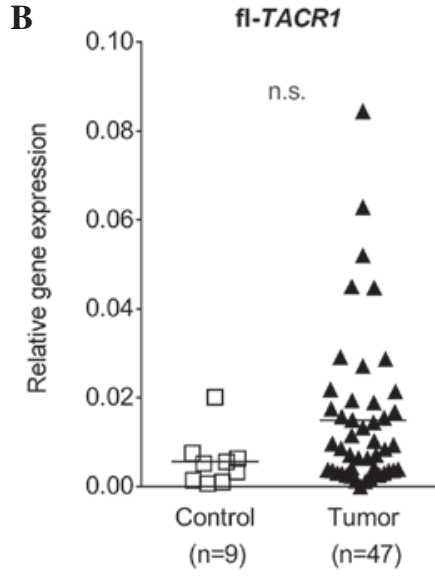

D

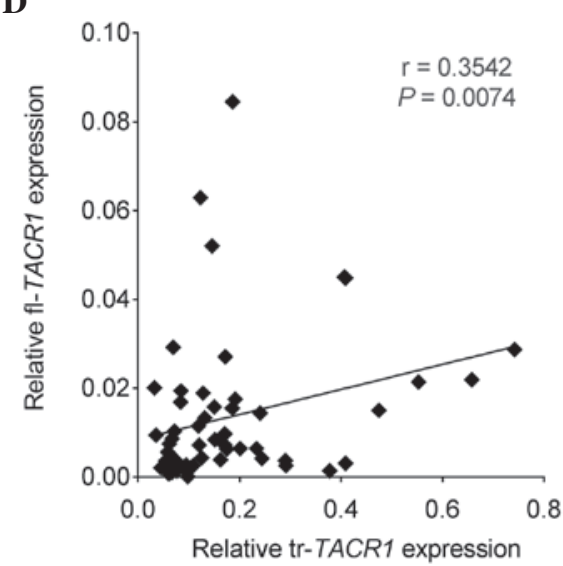

Figure 1. Hepatoblastoma tumors overexpress TACRl compared with normal liver tissue. (A) Statistically significant differences in the relative gene expression levels of tr-TACR1 in HB ( $\mathrm{n}=47$, black triangles) compared with normal liver tissue ( $\mathrm{n}=9$, white squares; $\mathrm{P}=0.0301)$. (B) No statistically significant differences in the gene expression levels of fl-TACR1 in the same samples as in (A). (C) Ratio of the gene expression values of (A) tr-TACR1 vs. (B) fl-TACR1, calculated for HB tumors and liver tissue samples. (D) Graphical representation of the correlation of (A) tr-TACR1 vs. (B) fl-TACR1 gene expression levels (black squares; $\mathrm{P}=0.0074, \mathrm{r}=0.3542$ ). tr/fl-TACR1, truncated/full-length-neurokinin-1 receptor; n.s., not significant.

Reverse transcription- $q P C R(R T-q P C R)$. According to the modified method of Bigioni et al, the prepared cDNA $(2 \mu \mathrm{l})$ was used in a PCR with specific primers, based on the common sequence of the TACRl (NK1R) human isoforms, which yield a 186-bp fragment (27). Specific primers were as follows: Forward, 5'-AACCCCATCATCTACTGCTGC-3' and reverse, 5'-ATTTCCAGCCCCTCATAGTCG-3' for fl-TACR1 (NM_001058.3); forward, 5'-GGGCCACAAGACCATCTA CA-3' and reverse, 5'-AAGTTAGCTGCAGTCCCCAC-3' for tr-TACR1 (NM_015727.2); and forward, 5'-GCCCGAAAC GCCGAATAT-3' and reverse, 5'-CCGTGGTTCGTGGCT CTCT-3' for the TBP housekeeping gene. The amplification reactions were performed with iTaq $S Y B R^{\circledR}$-Green Supermix (Bio-Rad Laboratories, Hercules, CA, USA) in a final reaction volume of $20 \mu \mathrm{l}$ and were incubated at $95^{\circ} \mathrm{C}$ for $7 \mathrm{~min}$, subjected to 40 cycles of $95^{\circ} \mathrm{C}$ for $30 \mathrm{sec}, 60^{\circ} \mathrm{C}$ for $30 \mathrm{sec}$ and $72^{\circ} \mathrm{C}$ for $30 \mathrm{sec}$, followed by a final extension cycle at $72^{\circ} \mathrm{C}$ for $7 \mathrm{~min}$. PCR was performed using a Mastercycler ep Gradient S (Eppendorf, Hamburg, Germany) and the transcript numbers were normalized according to the expression of the housekeeping gene. Relative quantification of gene expression was performed using the $2^{-\Delta \Delta \mathrm{Ct}}$ method, as described by Pfaffl (28).

Statistical analysis. Data are presented as mean \pm SD. Mean and individual relative expression values of tumor and control samples are expressed in dot plots for each group. Statistical comparisons were performed with a standard t-test and Mann-Whitney U test using GraphPad Prism biostatistics software (version 5.0d; GraphPad Software, Inc., La Jolla, CA, USA). $\mathrm{P}<0.05$ and $\mathrm{P}<0.01$ indicated a statistically significant difference for all the comparisons. To differentiate between a high and low expression of TACR 1 and its components, 3 -fold of the mean of 9 tumor-free control samples was used as a cutoff for high expression. Kaplan-Meier estimates of specific survival time in the two groups were compared using the log-rank Mantel-Cox test.

\section{Results}

TACRl is overexpressed in human HB patients. To address the aforementioned hypotheses, the gene expression pattern of fl-TACR 1 and tr-TACR 1 were analyzed in tumor tissue samples of $\mathrm{HB}$ and non-tumorous liver tissue. A significantly higher expression of tr-TACR 1 was observed in HB compared with the control specimens $(\mathrm{P}=0.0301$; Fig. 1A). Although not statistically significant, the expression of fl-TACR1 also tended to be higher in tumor specimens $(\mathrm{P}>0.05$; Fig. 1B). These results correlated with our previous findings, in which in vitro and in vivo models of $\mathrm{HB}$ were used to demonstrate that tr-TACRI is overexpressed in malignant $\mathrm{HB}$ cells. In turn, malignant HB 
A
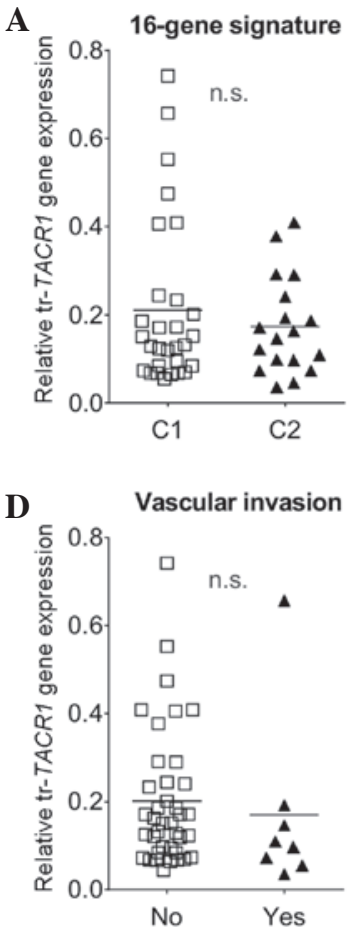

G

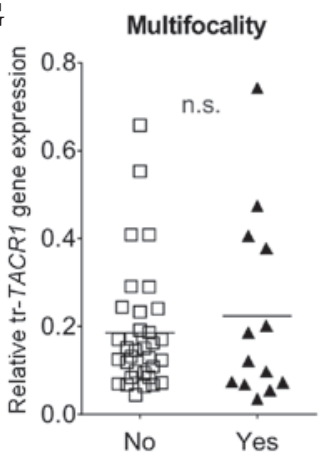

B

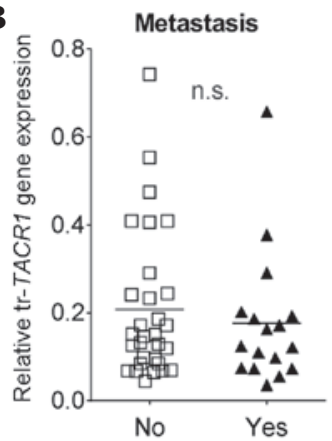

$\mathbf{E}$

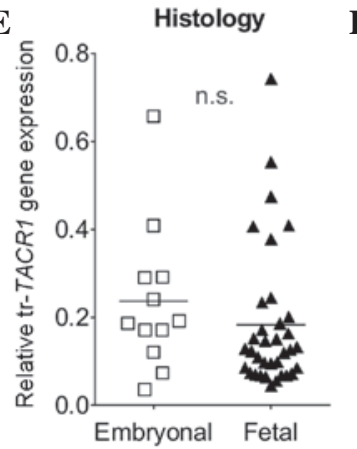

$\mathbf{H}$

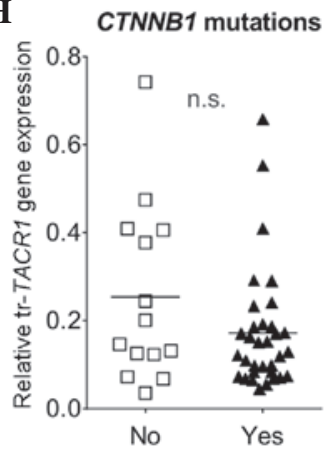

C

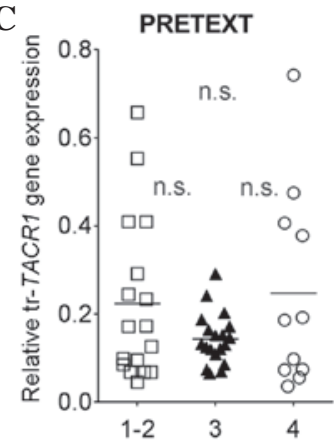

F

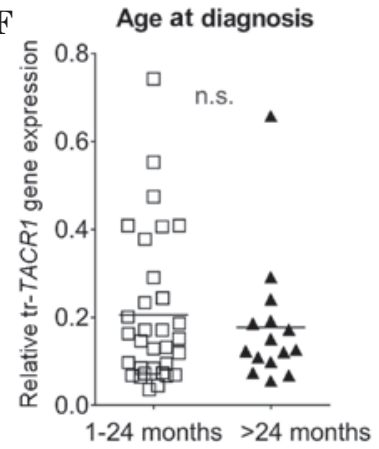

1-24 months $>24$ months

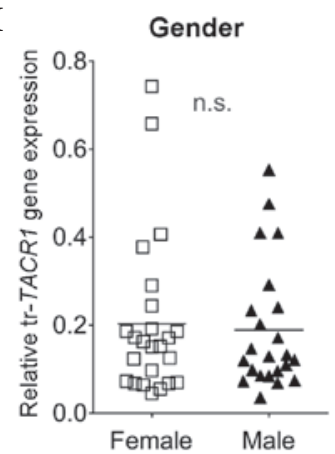

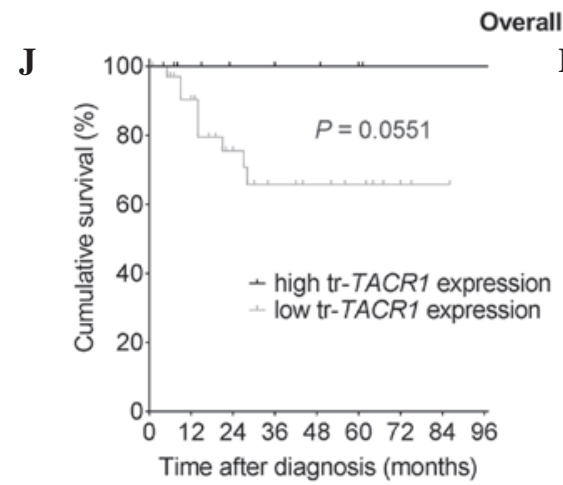

verall survival

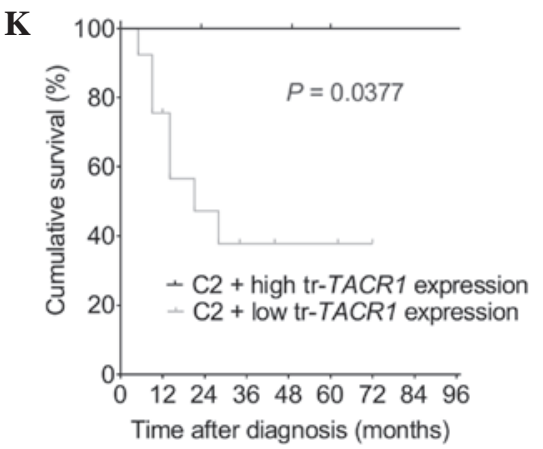

Figure 2. tr-TACR1 expression is not significantly associated with biological, clinical and histological parameters. Relative gene expression of tr-TACR1 was correlated to the (A) 16-gene signature, (B) metastasis, (C) the preoperative staging system PRETEXT, (D) vascular invasion, (E) histology, (F) age at diagnosis, (G) multifocality, (H) CTNNB1 mutation status (no represents wild-type, yes represents mutated $\beta$-catenin) and (I) gender; however, the differences were not significant $(\mathrm{P}>0.05)$. ( $\mathrm{J})$ Overall survival for high and low tr-TACR1 expression revealed a non-significant difference in survival ( $\mathrm{P}=0.0551)$. (K) Low expression of tr-TACR1 was associated with significantly lower overall survival in hepatoblastoma tumors harboring the $\mathrm{C} 2$ signature $(\mathrm{P}=0.0377)$. tr-TACR1, truncated-neurokinin-1 receptor; n.s., not significant.

cells were correlated with responsiveness to treatment with NK1R antagonists, such as aprepitant (8).

Expression of tr-TACRl correlates with fl-TACR1. Due to the wide range of gene expression, the ratio of tr-TACRl versus
fl-TACR 1 expression was presented. It was found that the ratios were comparable between the tumor and control samples (Fig. 1C), suggesting a positive correlation of the two splice variants. When analyzed in-depth, a statistically significant weak correlation was identified between tr-TACRl and 
Table I. Clinical, biological and histological outcome characteristics of 47 patients with hepatoblastoma.

\begin{tabular}{|c|c|}
\hline Characteristic & Patients, n (\% \\
\hline \multicolumn{2}{|c|}{ 16-gene signature } \\
\hline $\mathrm{C} 1$ & $29(61.7)$ \\
\hline $\mathrm{C} 2$ & $18(38.3)$ \\
\hline \multicolumn{2}{|l|}{ Metastasis } \\
\hline Yes & $17(36.2)$ \\
\hline No & $30(63.8)$ \\
\hline \multicolumn{2}{|l|}{ PRETEXT } \\
\hline I-II & $17(36.2)$ \\
\hline III & $19(40.4)$ \\
\hline IV & $11(23.4)$ \\
\hline \multicolumn{2}{|c|}{ Vascular Invasion } \\
\hline Yes & $8(17.1)$ \\
\hline No & $39(82.9)$ \\
\hline \multicolumn{2}{|l|}{ Histology } \\
\hline Fetal & $35(74.5)$ \\
\hline Embryonal & $12(25.5)$ \\
\hline \multicolumn{2}{|c|}{ Age at diagnosis, months } \\
\hline $1-24$ & $32(68.1)$ \\
\hline$>24$ & $15(31.9)$ \\
\hline \multicolumn{2}{|l|}{ Multifocality } \\
\hline Yes & $13(27.7)$ \\
\hline No & $34(72.3)$ \\
\hline \multicolumn{2}{|l|}{$C T N N B 1$ status } \\
\hline Wild-type & $14(29.8)$ \\
\hline Mutated & $33(70.2)$ \\
\hline \multicolumn{2}{|l|}{ Gender } \\
\hline Female & $24(51.1)$ \\
\hline Male & $23(48.9)$ \\
\hline
\end{tabular}

fl-TACRl expression $(\mathrm{r}=0.3542)$, potentially indicating a mutual dependency (Fig. 1D).

TACR1 expression does not correlate with biological characteristics. To improve understanding of whether splice variants or their ratio correlate with the biological features of the tumor, TACRI expression was analyzed accordingly (Table I; Figs. 2-4). First, the truncated variant was investigated, due to its significance in $\mathrm{HB}$ as a potential therapeutic target (8). In order to accomplish this, the relative expression of tr-TACR 1 was correlated with a recently described 16-gene molecular signature known to be associated with prognosis (16). Similar to the original description of this signature, the current cohort was separated into 29 patients with $\mathrm{HB}$ that exhibited the $\mathrm{C} 1$ signature (poor prognosis; 61.7\%) and 18 exhibited the $\mathrm{C} 2$ signature (improved prognosis; 38.3\%) (Table I). Relative gene expression analysis of tr-TACR 1 revealed no significant difference between patients with $\mathrm{C} 1$ and $\mathrm{C} 2$ signatures (Fig. 2A). The same features were then analyzed in correlation to the gene expression of fl-TACRl (Fig. 3A). A significant correlation was identified between low fl-TACRl expression and the C2 population of the 16 -gene signature $(\mathrm{P}=0.0222$; Fig. $4 \mathrm{~A})$. It is of note that $55.6 \%$ of the specimens grouped into the C2 population exhibited extremely low levels of fl-TACR1.

The ratio of fl-TACR 1 to tr-TACR 1 was then calculated to determine whether it could be correlated with the 16-gene signature (16). Notably, extremely low ratios were identified in the favorable $\mathrm{C} 1$ population and extremely high ratios were evident for the $\mathrm{C} 2$ population; however, this effect was not statistically significant (data not shown). Populations with a C2 signature were previously demonstrated to be associated with a poor prognosis of HB (16).

Expression of TACR1 does not correlate with clinical characteristics. The expression patterns of fl-TACRl and tr-TACRl were correlated with clinical, biological and histological characteristics, including metastasis (Figs. 2B and 3B), the preoperative classification PRETEXT (Figs. 2C and 3C), vascular invasion (Figs. 2D and 3D), histology (Figs. 2E and 3E), onset period (Figs. 2F and 3F), multifocality (Figs. $2 \mathrm{G}$ and $3 \mathrm{G}$ ), CTNNB1 mutations (Figs. 2H and 3H) and gender (Figs. 2I and 3I). Of the entire cohort, it was found that $63.8 \%$ exhibited no metastasis at the time of diagnosis, $82.9 \%$ had no vascular invasion and only $27.7 \%$ were multifocal. Gender was equally distributed (51.1\% female vs. $48.9 \%$ male), the majority tumors had a fetal histology (74.5 vs. $25.5 \%$ embryonal) and, as expected, $70.2 \%$ of tumors possessed a $\beta$-catenin (CTNNB1) mutation. The age of diagnosis was predominantly within the first 24 months of life $(68.1 \%)$ and the specimens were classified as PRETEXT 1-2 (36.2\%), PRETEXT 3 (40.4\%) and PRETEXT 4 (23.4\%).

When analyzing tr-TACRl expression in detail, no statistically relevant differences were identified with respect to the aforementioned clinical features. Notably, a high expression of tr-TACRl correlated with a better overall survival $(\mathrm{P}=0.0551$; Fig. 2J), although this was only a trend as it did not reach statistical significance.

Similarly, when analyzing the pattern of fl-TACR 1 expression with metastasis, PRETEXT, vascular invasion, histology, age at diagnosis, multifocality, CTNNB1 mutations or gender, no significant correlation was observed ( $P>0.05$; Fig. 3B-I). When clustered into groups of high versus low expression of fl-TACRl, overall survival curves did not deviate from each other $(\mathrm{P}>0.05$; Fig. 3J), contrary to the finding for tr-TACRl (Fig. 2J).

Use of the ratio of the two variants (tr-TACRl:fl-TACRl), revealed no statically significant differences with regard to the majority of the characteristics (Fig. 4A-I). The only exception to this was that a higher tr-TACRl:fl-TACR1 ratio, which occurred predominantly in PRETEXT 1-2 compared with PRETEXT 3 ( $\mathrm{P}=0.0459$; Fig. 4C). Similar to the analysis with tr-TACRl alone, overall survival was worse with a low ratio of tr-TACRl:fl-TACRl (P>0.05; Fig. 4J).

As the original description of the 16-gene signature by Cairo et al (16) suggested a worse prognosis for the C2 
A

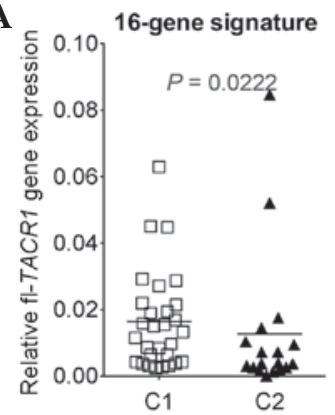

D

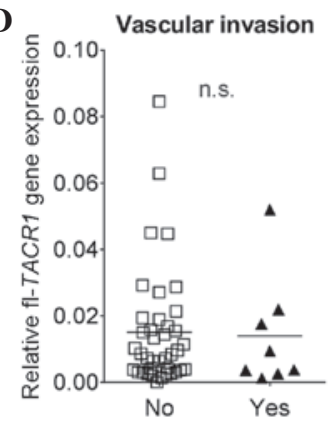

G

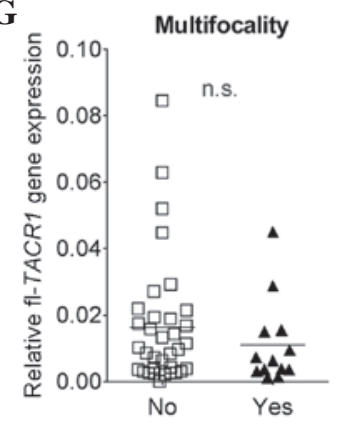

B

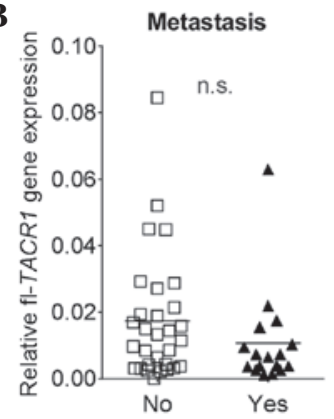

$\mathbf{E}$

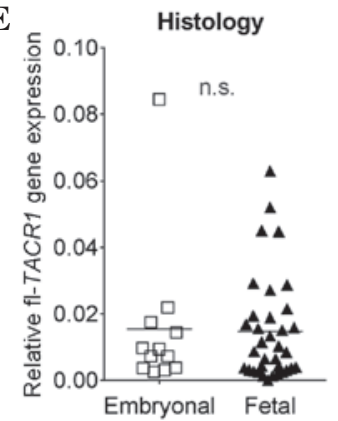

C

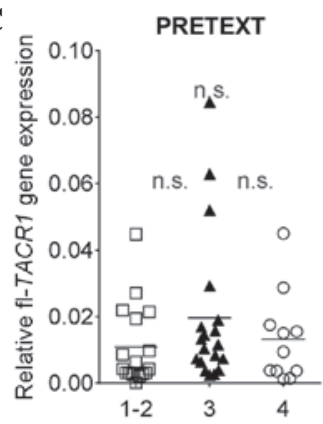

$\mathbf{F}$

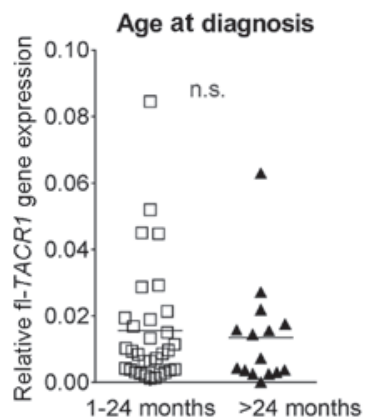

H

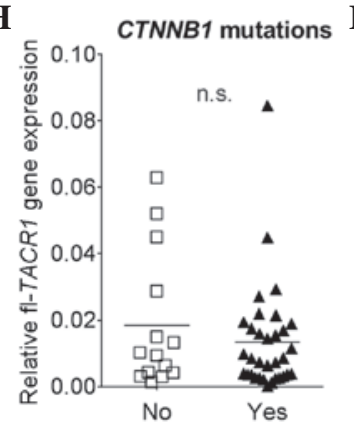

Gender

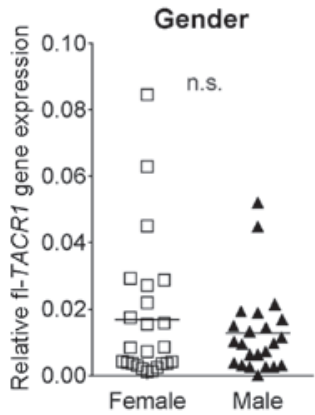

Overall survival
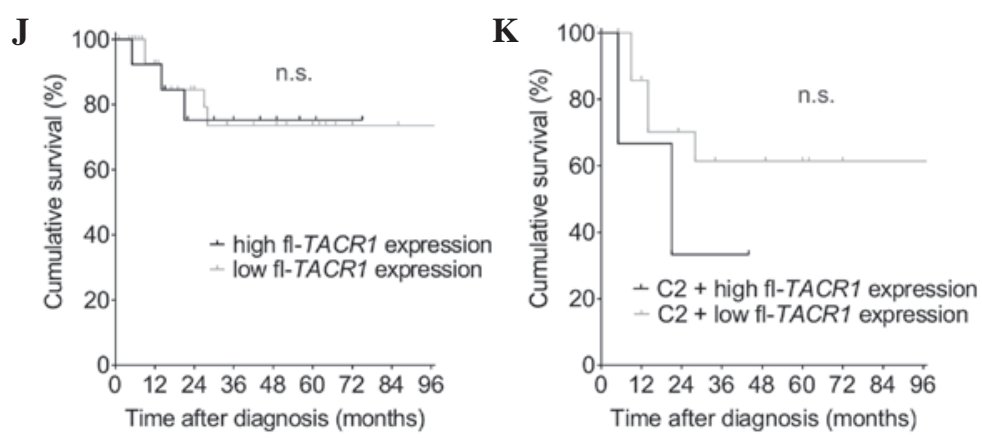

Figure 3. fl-TACR1 expression displays no significant difference in the majority of biological, clinical and histological features. (A-I) Analogous to Fig. 2, relative gene expression of fl-TACRl was compared with the same ten parameters. (A) The C2 signature significantly correlated with low expression of the fl-TACRl gene ( $\mathrm{P}=0.0222)$. P-values for (B) metastasis, (C) PRETEXT staging, (D) vascular invasion, (E) histology, (F) age at diagnosis, (G) multifocality, (H) CTNNB1 mutation status and (I) gender did not reveal any statistically significant differences $(\mathrm{P}>0.05)$. (J) Analysis of overall survival for high and low fl-TACR1 expression revealed no significant difference in survival $(\mathrm{P}>0.05)$. $(\mathrm{K})$ High expression of fl-TACR1 was associated with a worse outcome in hepatoblastoma tumors harboring the $\mathrm{C} 2$ signature, however, the trend was not significant $(\mathrm{P}>0.05)$. fl-TACR1, full-length neurokinin-1 receptor; n.s., not significant.

signature, the present study aimed to investigate whether either factor (tr-TACR1, fl-TACR1 or the ratio thereof) could refine the predictive value in our set of tumors. Therefore, overall survival within the C2 HB tumors was re-analyzed, and their outcome with respect to high versus low expression of TACRl or its ratio was investigated. It was identified that low tr-TACR1 predicted a poor prognosis for $\mathrm{C} 2$ tumors with a higher significance than tr-TACRl alone ( $\mathrm{P}=0.0377$; Fig. $2 \mathrm{~K})$.
Although not significant, high fl-TACR 1 suggested a worse outcome ( $\mathrm{P}>0.05$; Fig. $3 \mathrm{~K})$, and the ratio of the two variants had the same trend as truncated alone and as analyzed in the whole cohort, but with a clear tendency towards a worse prognosis for low tr/fl-TACR1 in the $\mathrm{C} 2$ group $(\mathrm{P}>0.05$; Fig. 4K).

Taken together, no strong correlation of tr-TACR 1 , fl-TACR I or tr-TACRl:fl-TACR I gene expression with clinical 

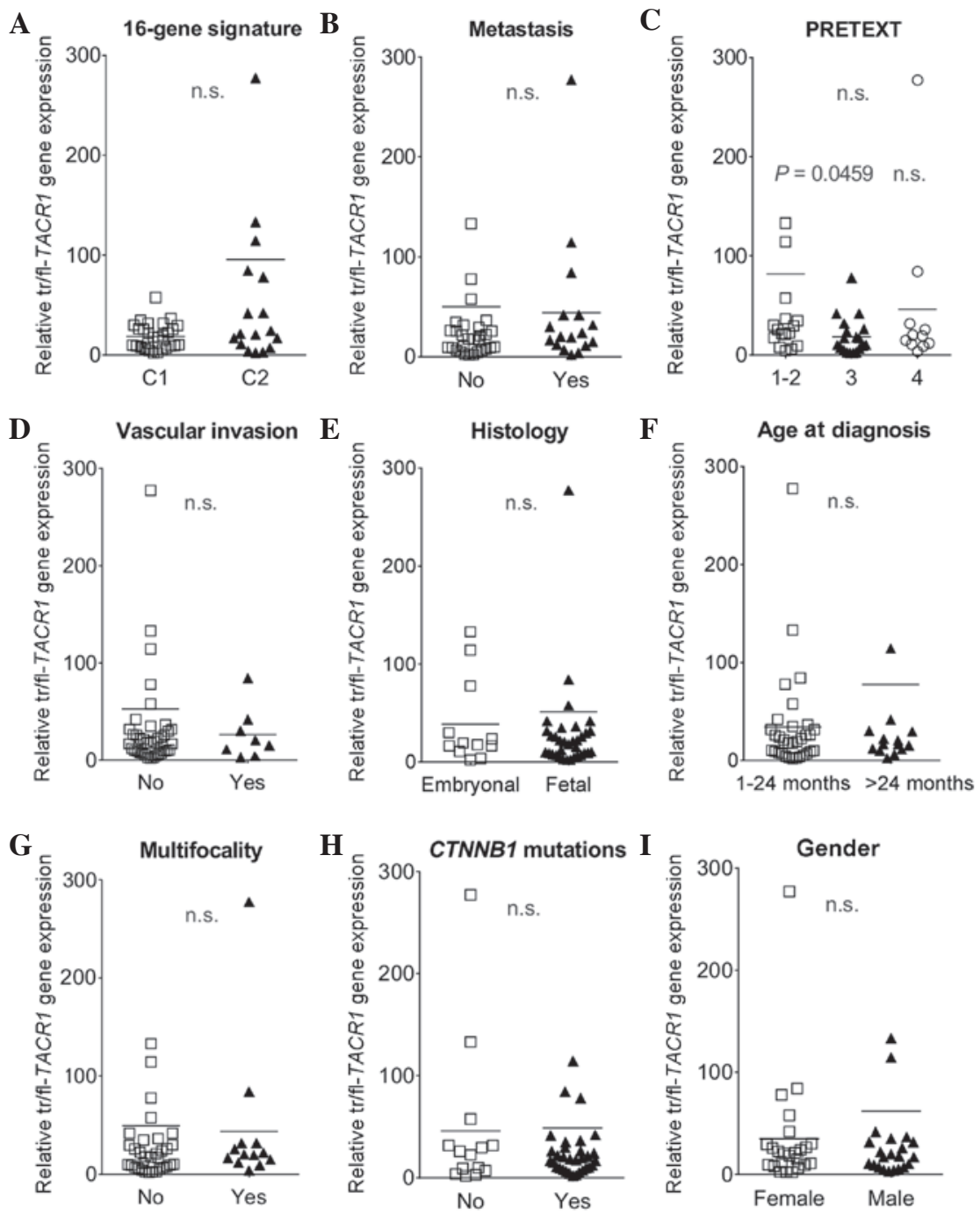

Overall survival
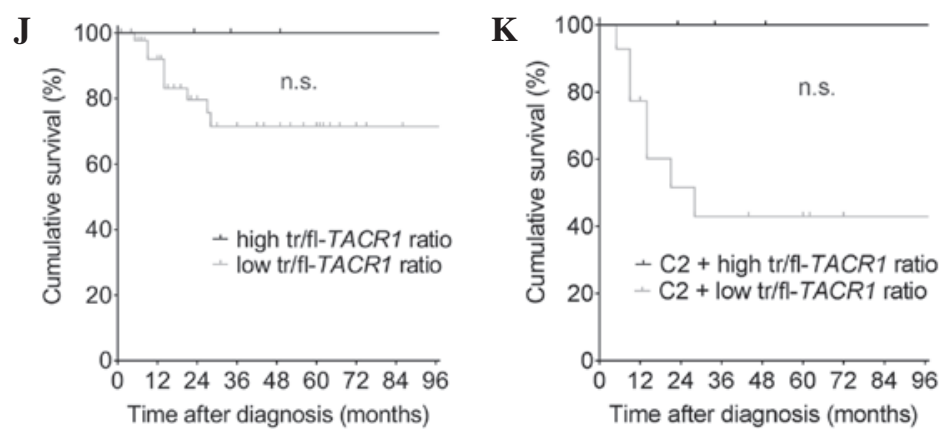

Figure 4. Ratio of tr-TACR1 and fl-TACR1 does not predict clinical prognosis. As in Figs. 2 and 3, ten different clinical features were analyzed with regard to the tr-TACR1:fl-TACR1 ratio gene expression. No significant difference were detected in the (A) 16-gene signature, (B) metastasis, (D) vascular invasion, (E) histology, (F) age at diagnosis, (G) multifocality, (H) CTNNB1 mutation status and (I) gender or (J) overall survival (P>0.05). (C) However, PRETEXT 1-2 significantly correlated with a higher expression ratio compared with PRETEXT 3 (P<0.05). (K) Low expression of the tr-TACRl:fl-TACR1 ratio was associated with lower overall survival in hepatoblastoma tumors harboring the $\mathrm{C} 2$ signature, however, the results were not significant $(\mathrm{P}<0.05)$. tr/fl-TACR1, truncated/full-length-neurokinin-1 receptor; n.s., not significant.

and histological data was identified. However, low tr-TACRI or a low ratio was associated with a worse prognosis, particularly when associated with the $\mathrm{C} 2$ signature. By contrast, no significance was identified in fl-TACR, with a marginal trend towards a worse outcome in $\mathrm{C} 2$ and high fl-TACRl expression.

\section{Discussion}

Little is known regarding the expression profile of TACRI and its associations with clinical outcome. NK1R is a crucial component of cancer development and progression. Thus, NK1R is a promising anticancer target in a multitude of cancer 
types, including $\mathrm{HB}(7,8)$. In the present study, in-depth analysis of the expression pattern of TACRl in HB was performed, and the findings were correlated with the patients' clinical tumor stage, biology and outcome. It was determined that, compared with tumor-free liver tissue, tumorous tissue expressed significantly more tr-TACR1. Although the difference was not significant, HB tissues also tended to express marginally more of fl-TACR 1 . This is in accordance with our recent description of this receptor in $\mathrm{HB}$ (8). Within the tumorous tissue, expression of fl-TACRl correlated with the expression of tr-TACRl. Furthermore, the expression of fl-TACRl was lower in the $\mathrm{C} 2$ (poor prognosis) compared with the $\mathrm{C} 1$ (improved prognosis) population of the 16-gene signature. When analyzing the expression of low versus high fl-TACRl within the $\mathrm{C} 2$ population only, no difference was found. There was also no correlation between tr-TACR 1 expression alone and any of the tumor characteristics investigated. However, a low expression of tr-TACRl demonstrated a clear trend towards worse prognosis but did not reach statistical significance. Thus, the current data provide evidence that HB ubiquitously expresses TACR1, supporting recent studies that NK1R antagonists may be promising anticancer agents against a wide variety $\mathrm{HB}$ subsets $(8,29)$.

It has previously been proposed that a correlation exists between the expression rate of the NK1R/SP complex and prognosis in various types of cancer (18-22). Garcia-Recio et al identified that SP contributes to persistent transmodulation of the ErbB receptors, epidermal growth factor receptor (EGFR) and human epidermal growth factor 2 (HER2), in breast cancer, acting to enhance malignancy and therapeutic resistance. Both TACR1 and TAC1 (SP) were highly expressed in HER2 ${ }^{+}$ primary breast tumors and correlated with poor prognosis factors (18). These findings are in contradiction to the current results in $\mathrm{HB}$, which indicated that worse prognosis was associated with a low expression of tr-TACR1. However, it should be noted that two separate tumor entities were investigated. In addition, Garcia-Recio et al (18) made no distinction between the truncated and the full-length variant of the receptor. Notably, following treatment of xenografted mice bearing HER $2^{+}$or HER2- human breast carcinoma, Garcia-Recio et al (18) only observed a therapeutic effect for HER $2^{+}$tumors, suggesting that the antitumor effects of NK1R inhibition in carcinoma of the breast depend on the modulatory properties of NK1R signaling on the activity of HER2 and EGFR (18).

In a different study, Gillespie et al identified that it was the expression of $\operatorname{tr}-T A C R 1$ and not fl-TACR 1 that predicted the progression from quiescent colitis to high-grade dysplasia and cancer in colitis-associated cancer (13). This is in accordance with the current results for $\mathrm{HB}$, in which tr-TACRl was observed to be upregulated in cancer cells but not in non-tumorous tissue.

Cairo et al recently described two tumor subclasses within $\mathrm{HB}$, resembling distinct phases of liver development and containing a discriminating 16-gene signature. Furthermore, it was found that $\beta$-catenin, a key protein of the Wnt signaling pathway, activated different transcriptional programs in the two distinct tumor subpopulations, $\mathrm{C} 1$ and $\mathrm{C} 2$. Notably, when separated into the two subpopulations by this 16-gene signature, clinical prognosis could be predicted for these children with an extremely high accuracy $(16,17)$. Considering these findings, the HB tumor bank was screened in the present study and each tumor was classified according to this specific 16-gene signature. Subsequently, the findings were correlated with the expression of fl-TACR 1 and tr-TACR 1 , and it was determined that fl-TACR 1 expression was lower in the $\mathrm{C} 2$ signature compared with the $\mathrm{C} 1$ group. A low expression of tr-TACR 1 was associated with a worse prognosis, although this was only a trend and not significant $(\mathrm{P}=0.0551)$. Of note, when analysis of the $\mathrm{C} 2$ signature population was performed separately, a low expression of tr-TACRl was significantly associated with a worse prognosis $(\mathrm{P}=0.0377)$. Therefore, it can be concluded that TACRl alone does not serve as a clinical marker for aggressiveness or potential to metastasize in HB. However, tr-TACR 1 may facilitate the identification of tumors that have a very poor prognosis, potentially alone but in particular within the $\mathrm{C} 2$ signature patient population. More in-depth analysis of such a $\mathrm{C} 2 \mathrm{tr}-T A C R 1^{\text {low }}$ tumor cohort is necessary to demonstrate the value of this distinction. Additionally, when making such distinction, it should be understood that 'low' expression in the present study is in reference to 'high' expression, as defined in the Patients and methods section. Thus, a tumor with tr-TACR $I^{\text {low }}$ expression may, on average, express significantly more tr-TACRl compared with non-tumorous tissue.

Previous studies identified that overexpression of tr-TACRI is associated with malignancy, including in $\mathrm{HB}(6-8,30)$. The present finding of low tr-TACR 1 correlating with worse prognosis initially appears to contradict this finding. Numerous reasons exist that possibly account for this discrepancy. One possible explanation is that tumors that express low levels of tr-TACR 1 represent an advanced stage in tumorigenesis characterized by profound immaturity, highlighted by the fact that a low expression of $\alpha$-fetoprotein (AFP), the only accepted tumor marker for HB, correlates with poor prognosis (1). It is proposed that this correlation occurs because tumor cells that are unable to produce AFP are more immature than AFP-producing tumor cells, leading to the recognized poor prognosis. However, this hypothesis cannot be adequately addressed by the data of the current study at this time.

Furthermore, tumors, and particularly tumors of the liver, have been shown to be significantly heterogeneous (31). Only one sample was analyzed per tumor in the current analysis, which may not be representative for other areas of the cancer. In addition, gene expression does not always correlate with protein expression. Therefore, it may be useful to determine whether an immunohistochemical (IHC) staining classification of HB may indicate more favorable clinical features and improved prognosis. However, IHC staining of the different splice variants of the NK1R/SP-complex remains a challenge and presents a major obstacle to this endeavor. Additionally, according to current understanding of the NK1R/SP-complex, $\mathrm{SP}$ is a critical ligand required for its function. The present study did not investigate the gene expression level of SP within the tumor or circulating protein of it; therefore, this should be performed in the future. Finally, all but four patients enrolled into this retrospective registry had received chemotherapy prior to surgery [8.8 vs. $27.7 \%$ in the original description of the 16-gene signature by Cairo et al(16)]. This is important to consider, as the exposure to chemotherapy could potentially alter the expression pattern of TACRI and its splice variants. Our previous study demonstrated that the expression of 
TACRl did not change with prior chemotherapy treatment in children with HB (8). However, these data did not distinguish between the full-length and the truncated version of the receptor (8). Therefore, the influence of systemic chemotherapy on the expression of the NK1R complex remains, to date, an unsolved question.

In conclusion, the results of the present study do not indicate that the TACRl expression pattern depends on or predicts the clinical stage and behavior of HB. However, two splice variants of TACRl were demonstrated to be ubiquitously overexpressed in HB. Furthermore, the current analysis suggests that the prediction of overall survival in the $\mathrm{C} 2$ signature-expressing $\mathrm{HB}$ subgroup may be refined by tr-TACR 1 and fl-TACR1, identifying a C2-TACR $1^{\text {low }}$ population with particularly poor prognosis. Overall, the present data further support the potential of the NK1R/SP complex as an ideal target in a wide variety of HB subsets.

\section{Acknowledgements}

We are grateful to Mrs. Fatemeh Promoli for technical assistance. Dr Michael Berger and Dr Matthias Ilmer were supported by postdoctoral stipends from the German Academic Exchange Program. Dr Michael Berger was additionally funded by the Friedrich-Baur Foundation Munich, Münchener Medizinische Wochenschrift, as well as the Funding for Research and Teaching of Ludwig Maximilian University of Munich. Professor Roland Kappler obtained funding from the Bettina Bräu Foundation (Munich, Germany) and the Gänseblümchen-Voerde Foundation (Voerde, Germany).

\section{References}

1. von Schweinitz D: Hepatoblastoma: Recent developments in research and treatment. Semin Pediatr Surg 21: 21-30, 2012.

2. Haeberle B and Schweinitz D: Treatment of hepatoblastoma in the German cooperative pediatric liver tumor studies. Front Biosci (Elite Ed) 4: 493-498, 2012.

3. Warmann S, Hunger M, Teichmann B, et al: The role of the MDR1 gene in the development of multidrug resistance in human hepatoblastoma: Clinical course and in vivo model. Cancer 95: 1795-1801, 2002.

4. Muñoz M, Rosso M and Coveñas R: The NK-1 receptor: A new target in cancer therapy. Curr Drug Targets 12: 909-921, 2011.

5. Palma C, Bigioni M, Irrissuto C, et al: Anti-tumour activity of tachykinin NK1 receptor antagonists on human glioma U373 MG xenograft. Br J Cancer 82: 480-487, 2000.

6. Rosso M, Muñoz M and Berger M: The role of neurokinin-1 receptor in the microenvironment of inflammation and cancer ScientificWorldJournal 2012: 381434, 2012.

7. Muñoz M, Rosso M and Coveñas R: A new frontier in the treatment of cancer: NK-1 receptor antagonists. Curr Med Chem 17: 504-516, 2010.

8. Berger M, Neth O, Ilmer M, et al: Hepatoblastoma cells express truncated neurokinin-1 receptor and can be growth inhibited by aprepitant in vitro and in vivo. J Hepatol 60: 985-994, 2014.

9. Muñoz M, Pérez A, Rosso M, Zamarriego C and Rosso R: Antitumoral action of the neurokinin-1 receptor antagonist L-733 060 on human melanoma cell lines. Melanoma Res 14: 183-188, 2004.

10. Mayordomo C, García-Recio S, Ametller E, et al: Targeting of substance $\mathrm{P}$ induces cancer cell death and decreases the steady state of EGFR and Her2. J Cell Physiol 227: 1358-1366, 2012.

11. Muñoz M, Berger M, Rosso M, et al: Antitumor activity of neurokinin-1 receptor antagonists in MG-63 human osteosarcoma xenografts. Int J Oncol 44: 137-146, 2014.

12. Kramer MS, Cutler N, Feighner J, et al: Distinct mechanism for antidepressant activity by blockade of central substance P receptors. Science 281: 1640-1645, 1998.
13. Gillespie E, Leeman SE, Watts LA, et al: Truncated neurokinin-1 receptor is increased in colonic epithelial cells from patients with colitis-associated cancer. Proc Natl Acad Sci USA 108: 17420-17425, 2011

14. Ramkissoon SH, Patel PS, Taborga M and Rameshwar P: Nuclear factor-kappaB is central to the expression of truncated neurokinin-1 receptor in breast cancer: Implication for breast cancer cell quiescence within bone marrow stroma. Cancer Res 67: 1653-1659, 2007.

15. Patel HJ, Ramkissoon SH, Patel PS and Rameshwar P: Transformation of breast cells by truncated neurokinin-1 receptor is secondary to activation by preprotachykinin-A peptides. Proc Natl Acad Sci USA 102: 17436-17441, 2005.

16. Cairo S, Armengol C, De Reyniès A, et al: Hepatic stem-like phenotype and interplay of Wnt/ $\beta$-catenin and Myc signaling in aggressive childhood liver cancer. Cancer Cell 14: 471-484, 2008.

17. Cairo S, Armengol C and Buendia MA: Activation of Wnt and Myc signaling in hepatoblastoma. Front Biosci (Elite Ed) 4: 480-486, 2012.

18. Garcia-Recio S, Fuster G, Fernandez-Nogueira P, et al: Substance $\mathrm{P}$ autocrine signaling contributes to persistent HER2 activation that drives malignant progression and drug resistance in breast cancer. Cancer Res 73: 6424-6434, 2013.

19. Esteban F, Muñoz M, González-Moles MA and Rosso M: A role for substance $\mathrm{P}$ in cancer promotion and progression: A mechanism to counteract intracellular death signals following oncogene activation or DNA damage. Cancer Metastasis Rev 25: 137-145, 2006.

20. Esteban F, Gonzalez-Moles MA, Castro D, et al: Expression of substance $\mathrm{P}$ and neurokinin-1-receptor in laryngeal cancer: Linking chronic inflammation to cancer promotion and progression. Histopathology 54: 258-260, 2009.

21. Castro TA, Cohen MC and Rameshwar P: The expression of neurokinin-1 and preprotachykinin-1 in breast cancer cells depends on the relative degree of invasive and metastatic potential. Clin Exp Metastasis 22: 621-628, 2005.

22. Misawa K, Kanazawa T, Misawa Y, et al: Frequent promoter hypermethylation of tachykinin-1 and tachykinin receptor type 1 is a potential biomarker for head and neck cancer. $J$ Cancer Res Clin Oncol 139: 879-889, 2013.

23. Pritchard J, Brown J, Shafford E, et al: Cisplatin, doxorubicin, and delayed surgery for childhood hepatoblastoma: A successful approach - results of the first prospective study of the International Society of Pediatric Oncology. J Clin Oncol 18: 3819-3828, 2000.

24. Roebuck DJ, Aronson D, Clapuyt P, et al; International Childrhood Liver Tumor Strategy Group: PRETEXT: A revised staging system for primary malignant liver tumours of childhood developed by the SIOPEL group. Pediatr Radiol 37: 123-132; quiz 249-150, 2007.

25. Eichenmüller M, Trippel F, Kreuder M, et al: The genomic landscape of hepatoblastoma and their progenies with HCC-like features. J Hepatol 61: 1312-1320, 2014.

26. Eichenmüller M, Gruner I, Hagl B, Häberle B, Müller-Höcker J, von Schweinitz D and Kappler R: Blocking the hedgehog pathway inhibits hepatoblastoma growth. Hepatology 49: 482-490, 2009.

27. Bigioni M, Benzo A, Irrissuto C, Maggi CA and Goso C: Role of NK-1 and NK-2 tachykinin receptor antagonism on the growth of human breast carcinoma cell line MDA-MB-231. Anticancer Drugs 16: 1083-1089, 2005.

28. Pfaffl MW: A new mathematical model for relative quantification in real-time RT-PCR. Nucleic Acids Res 29: e45, 2001.

29. Muñoz M and Coveñas R: Neurokinin-1 receptor: A new promising target in the treatment of cancer. Discov Med 10: 305-313, 2010.

30. Muñoz M and Rosso M: The NK-1 receptor antagonist aprepitant as a broad spectrum antitumor drug. Invest New Drugs 28: 187-193, 2010.

31. Nault JC and Villanueva A: Intratumor molecular and phenotypic diversity in hepatocellular carcinoma. Clin Cancer Res 10: 1786-1788, 2015. 